\title{
A nondestructive Bell-state measurement on two distant atomic qubits
}

\author{
Stephan Welte, Philip Thomas, Lukas Hartung, Severin Daiss, Stefan Langenfeld $\odot$, Olivier Morin $₫$, \\ Gerhard Rempe and Emanuele Distante ${ }^{\circ} \bowtie$
}

\begin{abstract}
One of the most fascinating aspects of quantum networks is their capability to distribute entanglement as a nonlocal communication resource'. In a first step, this requires network-ready devices that can generate and store entangled states $^{2}$. Another crucial step, however, is to develop measurement techniques that allow for entanglement detection. Demonstrations for different platforms ${ }^{3-13}$ suffer from being not complete, destructive or local. Here, we demonstrate a complete and nondestructive measurement scheme ${ }^{14-16}$ that always projects any initial state of two spatially separated network nodes onto a maximally entangled state. Each node consists of an atom trapped inside an optical resonator from which two photons are successively reflected. Polarization measurements on the photons discriminate between the four maximally entangled states. Remarkably, such states are not destroyed by our measurement. In the future, our technique might serve to probe the decay of entanglement and to stabilize it against dephasing via repeated measurements ${ }^{17,18}$.
\end{abstract}

Joint measurements that detect entangled states of multiple stationary qubits are a backbone for the development of quantum networks. One prominent example comprises Bell-state measurements (BSMs) that detect the maximally entangled Bell states (BSs) of two qubits $^{3}$. The BSMs enable fundamental protocols such as quantum teleportation and entanglement swapping ${ }^{19}$ for the purpose of quantum-information transfer and entanglement distribution over the network. A novel and fascinating scenario occurs for measurements that are able to detect an entangled BS without disturbing it. Such nondestructive BSMs ${ }^{14-16}$ project any state of the measured qubits onto the detected entangled state. This allows one to repeatedly measure a BS, thus opening up a route towards a new class of applications. Among others, an intriguing perspective is the possibility to protect the entangled state of two distant network qubits against environment-induced decoherence via the quantum Zeno effect $^{17,18}$. To this end, however, the measurement must be efficient and the detection time should be faster than the coherence time of the entangled state. Here, the entangled state coherence is assumed to decay more slowly than exponentially in time.

An ideal BSM should primarily be complete: it should be able to distinguish between all four BSs of two qubits, defined as $\left|\Phi^{ \pm}\right\rangle=\frac{1}{\sqrt{2}}\left(\left|\uparrow_{z} \uparrow_{z}\right\rangle \pm\left|\downarrow_{z} \downarrow_{z}\right\rangle\right)$ and $\left|\Psi^{ \pm}\right\rangle=\frac{1}{\sqrt{2}}\left(\left|\uparrow_{z} \downarrow_{z}\right\rangle \pm\left|\downarrow_{z} \uparrow_{z}\right\rangle\right)$, where we have used $\left\{\left|\uparrow_{z}\right\rangle,\left|\downarrow_{z}\right\rangle\right\}$ as the qubit computational basis. However, developing a BSM for distant stationary qubits that is, at the same time, complete and nondestructive poses great experimental challenges. For example, complete BSMs can be realized by making two qubits interact via a quantum gate before measuring each qubit separately ${ }^{4,5}$. Although challenging, this protocol can be applied to qubits located in distant network nodes, provided that a nonlocal quantum gate is available $e^{20-22}$. In both scenarios, however, such a scheme projects the qubits onto separable states and thereby destroys the entanglement while detecting it. Alternatively, a BSM can be implemented by entangling each qubit with one photon, interfering the two photons on a beam splitter and detecting them with single-photon detectors ${ }^{6-11}$. This is particularly convenient for qubits residing in separate nodes as the photons can travel in optical fibres. However, this scheme is intrinsically not complete as only two out of the four BSs can be revealed ${ }^{23}$. An upgrade of this that realizes a nondestructive BSM that is also complete would require a photon-photon quantum gate ${ }^{24,25}$; however, this is hard to implement experimentally.

In this Letter, we demonstrate a different protocol that does not rely on photon interference or on a photon-photon gate, but still realizes a complete and nondestructive BSM of two atomic qubits located at two nodes of an elementary quantum network. We use two ancillary photons that travel between the nodes in an optical-fibre link and interact sequentially with both atoms before being detected. Using suitable local qubit rotations, a single-photon state detection can distinguish between either the $\Phi$ and $\Psi$ or the + and - manifolds of the BSs (Fig. 1a). Two photons can then carry all the information to discriminate the four BSs. In essence, each photon implements a nondestructive parity measurement, and two successive parity measurements together with the qubit rotations realize the nondestructive BSM scheme described in refs. ${ }^{15,16}$. A similar protocol has been recently demonstrated on the IBM five-qubit quantum processor chip ${ }^{12}$. However, it relies on a stationary ancilla and is thus restricted to the measurement of qubits on the same chip. Conversely, our realization employs travelling photons, enabling the detection of entangled states of qubits located further apart. The only intrinsic limitation is the optical loss in the connecting fibre, which, at a suitable wavelength, can be small for up to a few kilometres. Remarkably, the measurement time is much shorter than the coherence time of the atomic qubits. In a regime of reduced optical losses, this would allow us to use our scheme to stabilize the entangled state of distant qubits. Furthermore, as photons can connect multiple nodes, the presented scheme can be readily scaled up to generate and detect multi-qubit entangled states embedded in quantum networks ${ }^{26}$.

Our experimental set-up is shown in Fig. 1b. The qubits are two single ${ }^{87} \mathrm{Rb}$ atoms located at nodes 1 and 2, respectively. They are physically separated by $21 \mathrm{~m}$ and connected by a 60 -m single-mode optical fibre. Each atom is trapped at the centre of a high-finesse $\left(F=6 \times 10^{4}\right)$ optical cavity. Both cavities are single-sided such that light impinging on the input mirror will be effectively reflected back with high probability. The qubit space is formed by the two atomic ground states $\left|\downarrow_{z}\right\rangle=\left|5 S_{1 / 2}, F=1, m_{\mathrm{F}}=1\right\rangle$ 
$\mathbf{a}$

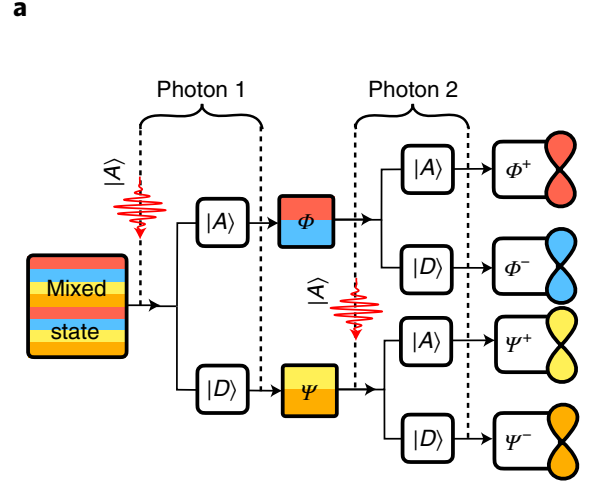

c

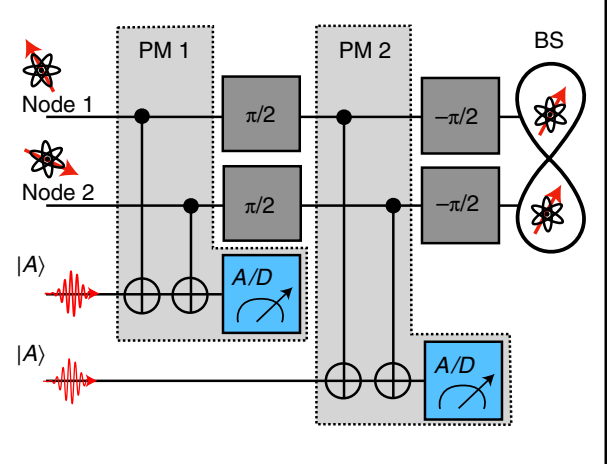

b

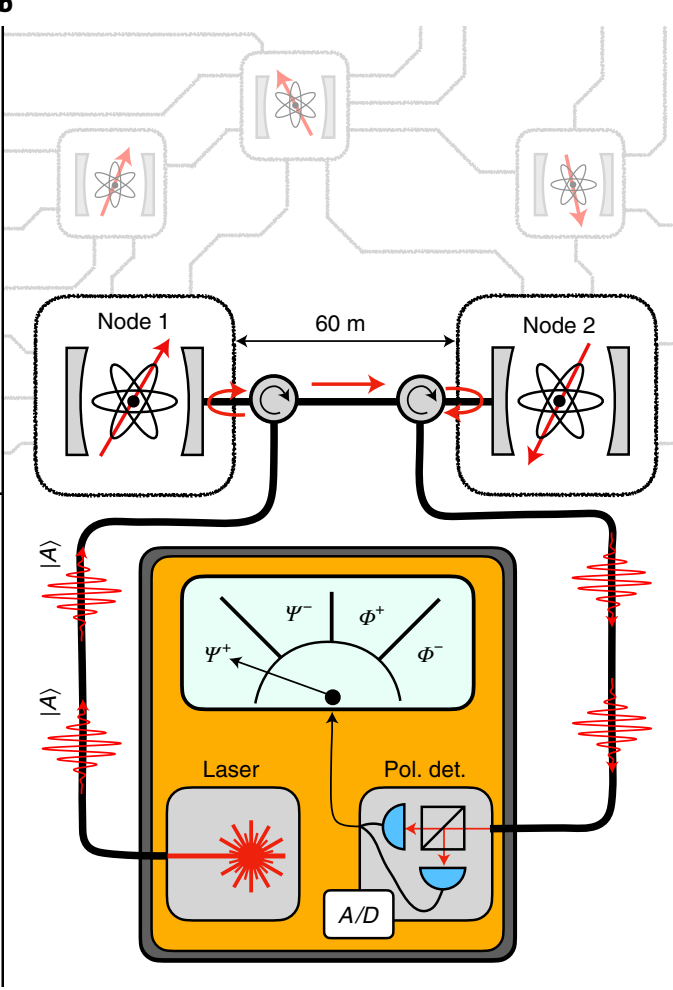

Fig. 1 | Set-up of the experiment. a, Decision tree to extract Bell states from a mixed state. Depending on the outcome of two photon-polarization measurements (antidiagonal $|A\rangle$ or diagonal $|D\rangle$ ), the four Bell states result. b, Our set-up comprises two single atoms (red arrows) trapped at the centre of two resonators located at node 1 and node 2 and connected by an optical fibre. This forms a simple quantum network link that could be part of a larger quantum network architecture with additional nodes (greyed out). The measurement device for the nondestructive BSM, depicted in yellow, consists of a laser and a polarization-sensitive set-up. It produces weak coherent pulses (red wiggly arrows) that are injected into the system, coupled to the resonators via optical circulators (depicted as circular arrows) and subsequently detected. Pol. det. indicates the polarization detection. c, Quantum circuit diagram of our protocol with two parity measurements (PM 1 and PM 2). Atom-photon gates are depicted as CNOT gates. The single-qubit rotations of the atoms are shown as grey boxes. The blue boxes represent the state detection of the two ancillary photons. The output Bell state (BS) is represented by the 8 -symbol.

and $\left|\uparrow_{z}\right\rangle=\left|5 S_{1 / 2}, F=2, m_{\mathrm{F}}=2\right\rangle$, and a pair of Raman lasers allows single-qubit rotations to be performed within this space. The cavities are actively stabilized to keep them resonant with the $\left|\uparrow_{z}\right\rangle \leftrightarrow|e\rangle=\left|5 P_{3 / 2}, F=3, m_{\mathrm{F}}=3\right\rangle$ atomic transition. The ancilla photons are polarization qubits at $780 \mathrm{~nm}$ resonant with the $\left|\uparrow_{z}\right\rangle \leftrightarrow|e\rangle$ transition. To approximate single photons, we use strongly attenuated Gaussian laser pulses of $1 \mu$ s duration (full-width at half-maximum, FWHM), with an average photon number $\bar{n} \ll 1$. At each node, an optical circulator is used to couple light to the resonator and collect the reflected signal. Each photon interacts first with the atom-cavity system at node 1, before being collected and routed via the optical fibre to node 2 . Here, it interacts with the second atom-cavity system and is finally guided to a photonic polarization-detection set-up realized with a combination of waveplates, a polarizing beam splitter and two superconducting nanowire single-photon detectors.

The main building block of our nondestructive BSM is a controlled-NOT (CNOT) gate between the atomic and the photonic qubit executed upon reflection of the photon from the resonator ${ }^{27,28}$. The gate relies on a specific light shift of the atomic energy levels engineered such that only right circularly polarized photons $|R\rangle$ couple to the atom (via the $\left|\uparrow_{z}\right\rangle \leftrightarrow|e\rangle$ transition), while left circularly polarized photons $|L\rangle$ do not. Because of the strong atom-cavity coupling, an atom in the coupled state $\left|\uparrow_{z}\right\rangle$ prevents an $|R\rangle$ photon from entering the cavity and thus the photon is directly reflected back from the first cavity mirror. By contrast, if the atom is in the uncoupled state $\left|\downarrow_{z}\right\rangle$ or the photon is $|L\rangle$ polarized, the light circulates in the cavity before being reflected back. This results in a $\pi$ phase shift of $\left|\uparrow_{z}, R\right\rangle$ relative to the cases $\left|\uparrow_{z}, L\right\rangle,\left|\downarrow_{z}, L\right\rangle,\left|\downarrow_{z}, R\right\rangle$. Such an atom-controlled $\pi$ phase shift realizes a CNOT gate in the linear polarization basis where an $\left|\uparrow_{z}\right\rangle$ atom flips an antidiagonal polarized $|A\rangle=1 / \sqrt{2}(i|R\rangle+|L\rangle)$ photon to its orthogonal diagonal polarization $|D\rangle=1 /(\sqrt{2} i)(i|R\rangle-|L\rangle)$ and vice versa, while the states $\left|\downarrow_{z}, D\right\rangle$ and $\left|\downarrow_{z}, A\right\rangle$ remain unchanged.

The quantum circuit diagram of our measurement scheme is shown in Fig. 1c. We employ antidiagonally polarized photons $|A\rangle$ that travel from node 1 to node 2 . This results in two successive atom-photon CNOT gates, which, together with the final photon polarization detection, realize a nondestructive parity measurement on the atoms, as indicated by the greyed box in Fig. 1c (details are provided in Supplementary Section 1). Specifically, a polarization detection in $|A\rangle$ projects the atoms on an even parity state, a linear combination of the atomic product states $\left|\downarrow_{z} \downarrow_{z}\right\rangle$ and $\left|\uparrow_{z} \uparrow_{z}\right\rangle$ that preserve the photon's polarization (here $|x y\rangle$ indicates state $|x\rangle$ and $|y\rangle$ on the first and second node, respectively). Conversely, a polarization detection in $|D\rangle$ projects the atoms onto an odd parity state, a linear combination of $\left|\uparrow_{z} \downarrow_{z}\right\rangle$ and $\left|\downarrow_{z} \uparrow_{z}\right\rangle$. As the BSs $\left|\Phi^{ \pm}\right\rangle$and $\left|\Psi^{ \pm}\right\rangle$ have opposite parity, a single ancilla is sufficient to distinguish between them. To discern between all four states, we apply two local $\pi / 2$ rotations to the atomic qubits after the first ancilla is detected. This effectively rotates $\left|\Phi^{-}\right\rangle$into $\left|\Psi^{+}\right\rangle$and vice versa, while leaving $\left|\Phi^{+}\right\rangle$and $\left|\Psi^{-}\right\rangle$unchanged (Supplementary Section 2). At this point, a second $|A\rangle$ polarized photon is employed to realize a second nondestructive parity measurement. There are four possible 
a
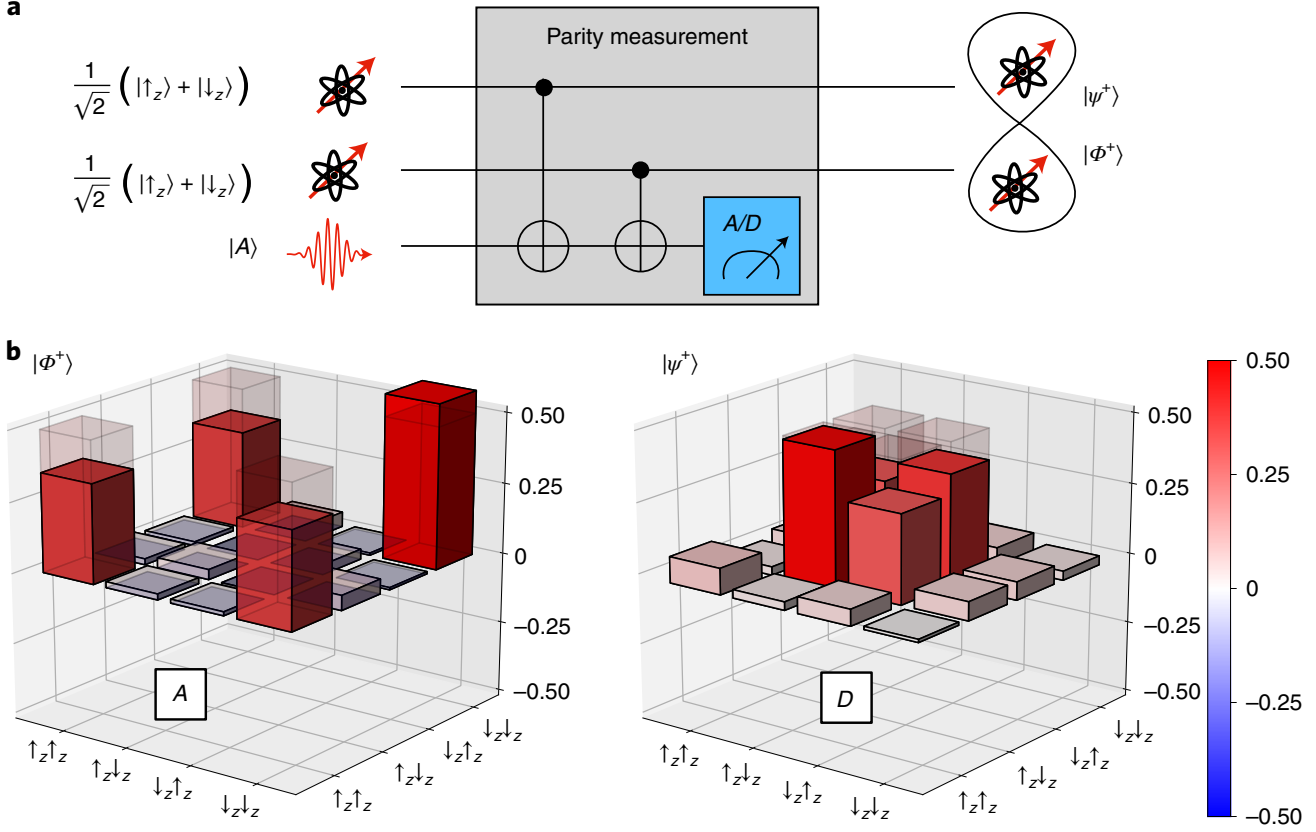

Fig. 2 | Nondestructive parity measurement. a, Quantum circuit diagram of the parity measurement. An antidiagonally polarized ancilla photon $|A\rangle$ reflects sequentially from node 1 and node 2 and implements a CNOT gate with each atomic qubit. The polarization detection on the ancilla projects the atoms on a state with known parity. For the shown initial state, this results in one of the entangled states $\left|\Phi^{+}\right\rangle$or $\left|\Psi^{+}\right\rangle$. b, Real part of the two-atom density matrices corresponding to the two possible measurement outcomes $(|A\rangle$ or $|D\rangle)$ of the photon polarization. The atoms are initially prepared in the state $1 / \sqrt{2}\left(\left|\uparrow_{z}\right\rangle+\left|\downarrow_{z}\right\rangle\right) \otimes 1 / \sqrt{2}\left(\left|\uparrow_{z}\right\rangle+\left|\downarrow_{z}\right\rangle\right)$, as indicated in $\mathbf{a}$. The two density matrices show a large overlap with the entangled states $\left|\Phi^{+}\right\rangle$and $\left|\Psi^{+}\right\rangle$ with fidelities $\mathcal{F}_{A}=(80.8 \pm 1.4) \%$ and $\mathcal{F}_{D}=(75.3 \pm 1.5) \%$, respectively (the errors indicate the standard deviation of the means).

outcomes of the two combined photon-polarization detections: $|A, A\rangle,|A, D\rangle,|D, A\rangle$ and $|D, D\rangle$, where the first (second) state in the ket indicates the detection result of the first (second) photon. These outcomes unambiguously identify $\left|\Phi^{+}\right\rangle,\left|\Phi^{-}\right\rangle,\left|\Psi^{+}\right\rangle$and $\left|\Psi^{-}\right\rangle$, respectively. Importantly, the atomic qubits are projected onto a known and still available entangled state, as only the two ancillary photons have been measured. At the end of the protocol, the two $\pi / 2$ pulses are reversed, with two additional $-\pi / 2$ rotations on each atomic qubit.

We first show that detecting the polarization of a single ancilla photon nondestructively measures the parity of the atomic state. To this end, we employ a coherent photon pulse with $\bar{n}=0.1$ and prepare the atomic qubits in a specific initial state $|\phi\rangle=1 / \sqrt{2}\left(\left|\uparrow_{z}\right\rangle+\left|\downarrow_{z}\right\rangle\right) \otimes 1 / \sqrt{2}\left(\left|\uparrow_{z}\right\rangle+\left|\downarrow_{z}\right\rangle\right)$, as shown in Fig. 2a. Because $|\phi\rangle=1 / \sqrt{2}\left(\left|\Psi^{+}\right\rangle+\left|\Phi^{+}\right\rangle\right)$, a nondestructive parity measurement should always project the atomic qubits on an entangled state, either $\left|\Phi^{+}\right\rangle$or $\left|\Psi^{+}\right\rangle$depending on whether $|A\rangle$ or $|D\rangle$ has been detected. We verify this by performing a full state tomography on the two atomic qubits, conditioned on a specific polarization detection. Our results are presented in Fig. $2 b$, where we show the reconstructed density matrices of the two possible final states. The measured fidelities with the ideal Bell states are $\mathcal{F}_{A}\left(\Phi^{+}\right)=(80.8 \pm 1.4) \%$ and $\mathcal{F}_{D}\left(\Psi^{+}\right)=(75.3 \pm 1.5) \%$ for a polarization detection in $|A\rangle$ and $|D\rangle$, respectively. Here and in the rest of this Letter, we define the fidelity $\mathcal{F}(x)$ of state $\rho$ with a pure state $|x\rangle$ as $\mathcal{F}(x)=\langle x|\rho| x\rangle$, and the errors indicate the standard deviation of the means. The experimental limitations in this experiment are discussed further in the following. The measurement demonstrates that one photon polarization detection distinguishes between even and odd parity states and thus between the $\Psi$ and $\Phi$ manifolds of the BSs.

As a next step, we show the full protocol of the nondestructive BSM. We start by demonstrating a specific feature of such a measurement, which is that any state of the two atoms is projected onto the detected BSs, and thus our BSM always generates entanglement. To this end, we prepare the system in the highly mixed state shown in Fig. $3 \mathrm{a}$. It is produced by randomly preparing each atom in one of six initial states $\left|\uparrow_{z}\right\rangle,\left|\downarrow_{z}\right\rangle,\left|\uparrow_{x}\right\rangle=\frac{1}{\sqrt{2}}\left(\left|\uparrow_{z}\right\rangle+\left|\downarrow_{z}\right\rangle\right),\left|\downarrow_{x}\right\rangle=\frac{1}{\sqrt{2}}\left(\left|\uparrow_{z}\right\rangle-\left|\downarrow_{z}\right\rangle\right)$, $\left|\uparrow_{y}\right\rangle=\frac{1}{\sqrt{2}}\left(\left|\uparrow_{z}\right\rangle+i\left|\downarrow_{z}\right\rangle\right)$ and $\left|\downarrow_{y}\right\rangle=\frac{1}{\sqrt{2}}\left(\left|\uparrow_{z}\right\rangle-i\left|\downarrow_{z}\right\rangle\right)$, where each state has equal probability to occur. We now perform the nondestructive BSM with two ancilla photon pulses that are both $|A\rangle$ polarized. We use an average photon number of $\bar{n}=0.34$ for each pulse, where the choice of a higher $\bar{n}$ allows the success probability of the final photon detections to increase. Conditioned on the four possible polarization-measurement outcomes $|A, A\rangle,|A, D\rangle,|D, A\rangle$ and $|D, D\rangle$, we perform a full state tomography on the two stationary qubits. The real parts of the reconstructed density matrices are reported in Fig. $3 b$ (the imaginary part is shown in Supplementary Fig. 2). For each detection outcome $|i, j\rangle$, we compute the fidelity $\mathcal{F}_{i, j}(x)$ of the atomic qubits state with the expected entangled state $x$. We find them to be $\mathcal{F}_{A, A}\left(\Phi^{+}\right)=(65.3 \pm 2) \%, \mathcal{F}_{A, D}\left(\Phi^{-}\right)=(68.8 \pm 2) \%$, $\mathcal{F}_{D, A}\left(\Psi^{+}\right)=(66.4 \pm 2) \%$ and $\mathcal{F}_{D, D}\left(\Psi^{-}\right)=(67.3 \pm 2) \%$. Thisyields an average fidelity of $\overline{\mathcal{F}}=(66.9 \pm 2 \%)$, which is substantially larger than the classical threshold of $50 \%$, thus certifying the genuine generation of entanglement. Our data show not only that the atomic qubits are always projected onto an entangled state, but also that each combination of polarization detection events is correlated with a different BS. Consequently, the two polarization measurements can be used to discriminate unambiguously between the four BSs. Remarkably, the time between the first photon ancilla and the detection of the second ancilla is $9 \mu$ s, substantially shorter than the measured atomic coherence time of $\sim 400 \mu$ s for each atom ${ }^{22}$.

Finally, to unambiguously demonstrate the nondestructive character of our BSM, we perform a complete tomography measurement ${ }^{29}$. Specifically, we reconstruct the four operators $\left\{\Pi_{j}\right\}$ that constitute the positive operator-valued measure (POVM) that fully describes our measurement. Here $j \in\{A A, A D, D A$, $D D\}$ is an index that labels the four possible outcomes of the 

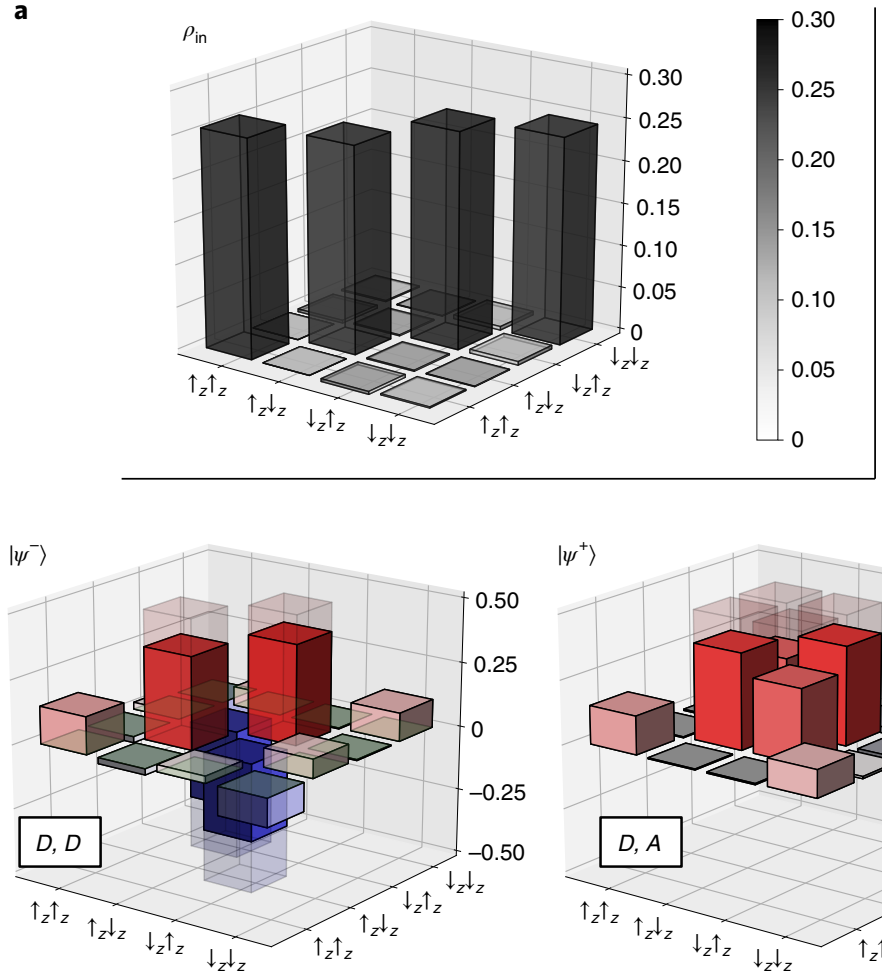

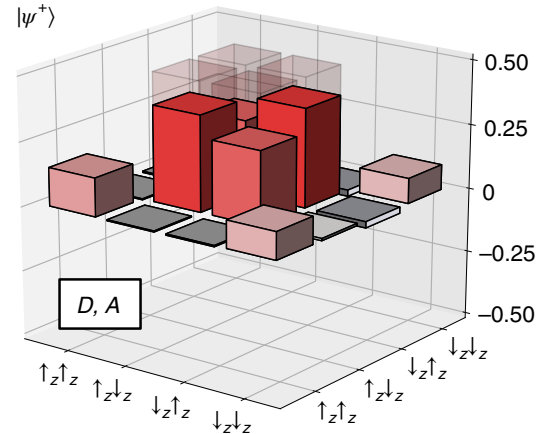

b

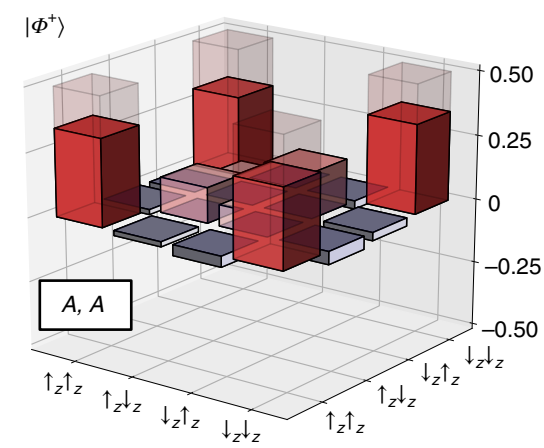

$\left|\Phi^{-}\right\rangle$

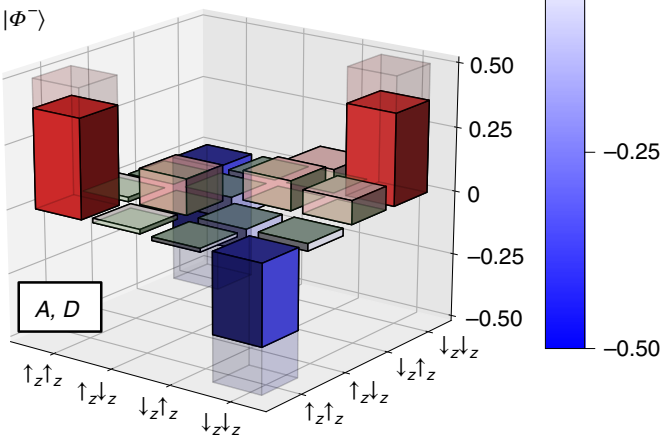

Fig. 3 | Deterministic generation of entanglement from a mixed state. a, Reconstructed density matrix of the fully mixed input state. $\mathbf{b}$, Real part of the reconstructed density matrices corresponding to the four possible measurement outcomes $|A, A\rangle,|A, D\rangle,|D, A\rangle$ and $|D, D\rangle$ of the two ancilla photons. Each density matrix shows a large overlap with one of the four BSs with fidelities $\mathcal{F}_{A, A}\left(\Phi^{+}\right)=(65.3 \pm 2) \%, \mathcal{F}_{A, D}\left(\Phi^{-}\right)=(68.8 \pm 2) \%$, $\mathcal{F}_{D, A}\left(\Psi^{+}\right)=(66.4 \pm 2) \%$ and $\mathcal{F}_{D, D}\left(\Psi^{-}\right)=(67.3 \pm 2) \%$. The errors indicate the standard deviation of the means. The transparent bars indicate the density matrix of the ideal BSs.

two polarization measurements. For an ideal BSM, each $\Pi_{j}$ would correspond to a projector onto one of the four BSs, explicitly $\left\{\Pi_{j}\right\}=\left\{\left|\Phi^{+}\right\rangle\left\langle\Phi^{+}|,| \Phi^{-}\right\rangle\left\langle\Phi^{-}|,| \Psi^{+}\right\rangle\left\langle\Psi^{+}|,| \Psi^{-}\right\rangle\left\langle\Psi^{-}\right|\right\}$. Following the quantum theory of measurement, for an initial density matrix $\rho$ of the two atomic qubits, the probability to detect an outcome $j$ is given by ${ }^{29}$

$$
p_{j}=\operatorname{Tr}\left[\rho \Pi_{j}\right]
$$

To reconstruct $\left\{\Pi_{j}\right\}$, we prepare different combinations of initial states with each atomic qubit in one of the six states $\left|\uparrow_{z}\right\rangle,\left|\downarrow_{z}\right\rangle$, $\left|\uparrow_{x}\right\rangle,\left|\downarrow_{x}\right\rangle,\left|\uparrow_{y}\right\rangle,\left|\downarrow_{y}\right\rangle$. This results in a total of 36 possible initial states of the two atoms. For each of these states, we run our BSM protocol and measure the probabilities $p_{j}$. In this way we obtain the full information to invert the relation given by equation (1) and to reconstruct the POVM $\left\{\Pi_{j}\right\}$. In Fig. 4, we show the real parts of the four reconstructed operators $\Pi_{j}$ in the Bell basis (the imaginary part is shown in Supplementary Fig. 3). Our data show that each $\Pi_{j}$ has a large overlap with one of the four BSs. The measured fidelities are $\mathcal{F}_{A A}\left(\Phi^{+}\right)=(61.7 \pm 1.9) \%, \quad \mathcal{F}_{A D}\left(\Phi^{-}\right)=(62.1 \pm 1.9) \%$, $\mathcal{F}_{D A}\left(\Psi^{+}\right)=(62.7 \pm 1.9) \%$ and $\mathcal{F}_{D D}\left(\Psi^{-}\right)=(62.7 \pm 1.9) \%$, where we have used the notation $\mathcal{F}_{j}(x)=\left\langle x\left|\Pi_{j}\right| x\right\rangle$. These data demonstrate that the polarization measurement of the ancillary photons is effectively equivalent to a measurement projecting on the Bell basis of the atomic qubits.

We numerically simulated our experiment including all known experimental imperfections (Supplementary Section 3). From the simulation we deduce the impact of the different imperfections on the measured fidelities. We identify two major contributions. First, the multi-photon component of the weak coherent pulses limits the underlying atom-photon CNOT gates ${ }^{28}$. This causes an overall fidelity loss of $4.3 \%$ and $9.8 \%$ for the data in Figs. 2 and 3, respectively. Second, the limited overlap of the cavity transversal modes and the mode of the fibres used to couple the ancilla photons in and out at both nodes gives a reduction of $5.1 \%$. The remaining contributions include atomic state preparation and measurement, preservation of polarization in the optical link, atomic state coherence and the limited coherent coupling of the atom to the cavity field compared to the dissipative processes in the system (losses of the resonator mirrors and atomic scattering rates). These are discussed in more detail in Supplementary Section 4 . We attribute the difference in the fidelities reported in Fig. 4 with respect to Fig. 3 to larger errors related to the preparation of the different atomic input states.

Unlike all previously implemented $\mathrm{BSMs}^{3-13}$, the complete, nonlocal and nondestructive nature of the presented scheme might allow the quantum Zeno effect to be used ${ }^{17,18}$ as a means to suppress the decoherence of any BS of two distant qubits. Explicitly, if the coherence of the entangled state decays more slowly than exponentially in time, repeated applications of our nondestructive BSM would continuously project the qubits with high probability to their initial entangled state, effectively evading the detrimental effect of the environment by freezing the quantum-state evolution. However, the BSM must be efficient and faster than the characteristic decay time of the entanglement. In our current implementation, the efficiency is limited to $0.1 \%$, as it is strongly affected by the overall optical losses ( $8.6 \%$ transmission from before the resonator at node 1 to a detected photon click) and the large vacuum component $(72 \%)$ of the employed weak coherent state. Future improved set-ups could strongly mitigate optical losses, including detector 

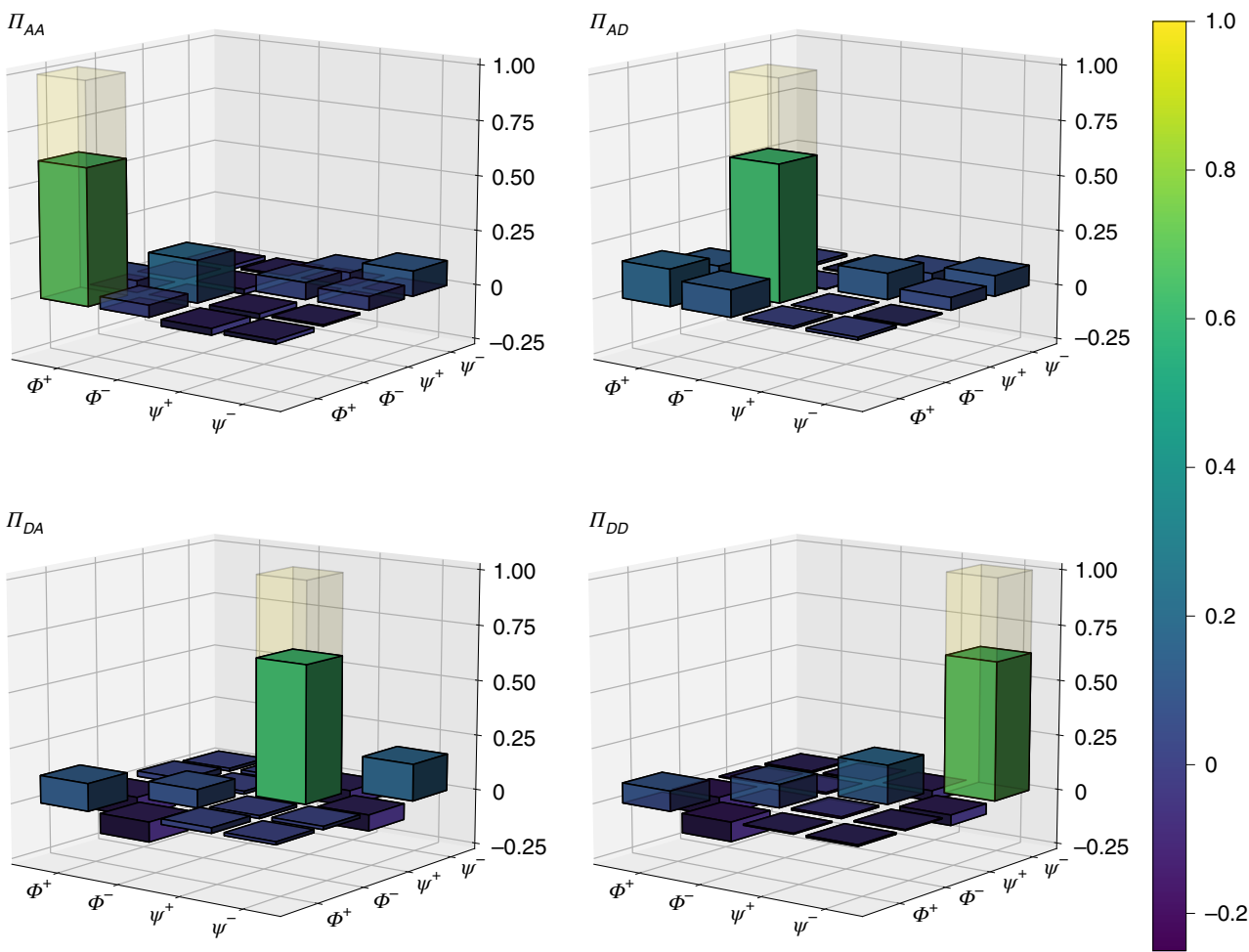

Fig. 4 | Measurement tomography. Real part of the reconstructed matrices describing the four operators $\left\{\Pi_{i}\right\}$ that constitute the POVM describing our measurement. The subscript $j \in\{A A, A D, D A, D D\}$ indicates the four possible outcomes of the polarization detection. The operator matrices are represented in the Bell basis $\left\{\left|\Phi^{ \pm}\right\rangle,\left|\Psi^{ \pm}\right\rangle\right\}$. The transparent bars represent the density matrices for the ideal POVM of a nondestructive BSM.

inefficiencies. This will enable use of an optimized sequence to render the scheme highly efficient, even with weak coherent states. In this case, the first pulse could be repeatedly sent until a successful photon detection occurs, because near-zero optical losses ensure that no photon has interacted with the qubits unless one is detected. The unitary $\pi / 2$ atomic rotation would then follow, after which a second series of pulses would be sent until another photon-detection event occurs. Assuming $\bar{n}=0.1$ per pulse and $4 \mu \mathrm{s}$ of atomic rotation (Methods), a successful measurement could be carried out in $24 \mu$ s on average, considerably shorter than our atomic coherence time. This could even be pushed down to $3 \mu \mathrm{s}$ using a deterministic single-photon source and faster atomic qubit rotations ${ }^{24}$.

A future implementation of our scheme could also improve on our reported fidelities, as they do not suffer from any fundamental limitation. In fact, by using a single-photon source, optimizing the cavity-to-fibre transversal mode matching and suppressing the polarization errors, the current average entanglement fidelity could be boosted from $\overline{\mathcal{F}}=67.9 \%$ to $\overline{\mathcal{F}} \approx 90 \%$. Further improvements are then possible by better controlling the atomic qubit state and reducing the fluctuations of the resonator frequencies.

Finally, a fascinating avenue is to extend the described nondestructive BSM to more nodes ${ }^{1,2}$. This could be straightforwardly realized as our implementation makes use of travelling photons that can connect many distant qubits to implement a multi-qubit parity measurement. Together with single-qubit rotations, this would enable the generation and detection of multi-qubit entangled Greenberger-Horne-Zeilinger (GHZ) states ${ }^{26}$ in a larger quantum network. Finally, we stress that the roles of the photonic ancilla and the atomic qubits are interchangeable. A slight modification to the current experiment can therefore be used to build a nondestructive BSM for photons. This could also be extended to generate and detect multi-photon GHZ states $^{30}$.

\section{Online content}

Any methods, additional references, Nature Research reporting summaries, source data, extended data, supplementary information, acknowledgements, peer review information; details of author contributions and competing interests; and statements of data and code availability are available at https://doi.org/10.1038/ s41566-021-00802-1.

\section{Received: 2 February 2021; Accepted: 21 March 2021;}

Published online: 3 May 2021

\section{References}

1. Wehner, S., Elkouss, D. \& Hanson, R. Quantum internet: a vision for the road ahead. Science 362, eaam9288 (2018).

2. Kimble, H. J. The quantum internet. Nature 453, 1023-1030 (2008)

3. Michler, M., Mattle, K., Weinfurter, H. \& Zeilinger, A. Interferometric Bell-state analysis. Phys. Rev. A 53, R1209-R1212 (1996).

4. Riebe, M. et al. Deterministic quantum teleportation with atoms. Nature 429, 734-737 (2004).

5. Barrett, M. D. et al. Deterministic quantum teleportation of atomic qubits. Nature 429, 737-739 (2004).

6. Chou, C. W. et al. Measurement-induced entanglement for excitation stored in remote atomic ensembles. Nature 438, 828-832 (2005).

7. Moehring, D. L. et al. Entanglement of single-atom quantum bits at a distance. Nature 449, 68-71 (2007).

8. Hofmann, J. et al. Heralded entanglement between widely separated atoms. Science 337, 72-75 (2012).

9. Nölleke, C. et al. Efficient teleportation between remote single-atom quantum memories. Phys. Rev. Lett. 110, 140403 (2013).

10. Bernien, H. et al. Heralded entanglement between solid-state qubits separated by three metres. Nature 497, 86-90 (2013).

11. Delteil, A. et al. Generation of heralded entanglement between distant hole spins. Nat. Phys. 12, 218-223 (2016).

12. Sisodia, M., Shukla, A. \& Pathak, A. Experimental realization of nondestructive discrimination of Bell states using a five-qubit quantum computer. Phys. Lett. A 381, 3860-3874 (2017). 
13. Stárek, R. et al. Nondestructive detector for exchange symmetry of photonic qubits. Npj Quantum Inf. 4, 1-7 (2018).

14. Barrett, S. D. et al. Symmetry analyzer for nondestructive Bell-state detection using weak nonlinearities. Phys. Rev. A 71, 060302 (2005).

15. Gupta, M., Pathak, A., Srikanth, R. \& Panigrahi, K. General circuits for indirecting and distributing measurement in quantum computation. Int. J. Quantum Inf. 5, 627-640 (2007).

16. Ionicioiu, R. Entangling spins by measuring charge: a parity-gate toolbox. Phys. Rev. A 75, 032339 (2007).

17. Misra, B. \& Sudarshan, E. C. The Zeno's paradox in quantum theory. J. Math. Phys. 18, 756-763 (1977).

18. Facchi, P. \& Pascazio, S. Quantum Zeno dynamics: mathematical and physical aspects. J. Phys. A 41, 493001 (2008).

19. Pirandola, S., Eisert, J., Weedbrook, C., Furusawa, A. \& Braunstein, S. L. Advances in quantum teleportation. Nat. Photon. 9, 641-652 (2015).

20. Chou, K. S. et al. Deterministic teleportation of a quantum gate between two logical qubits. Nature 561, 368-373 (2018).

21. Wan, Y. et al. Quantum gate teleportation between separated qubits in a trapped-ion processor. Science 364, 875-878 (2019)

22. Daiss, S. et al. A quantum-logic gate between distant quantum-network modules. Science 371, 614-617 (2021).

23. Lütkenhaus, N., Calsamiglia, J. \& Suominen, K. A. Bell measurements for teleportation. Phys. Rev. A 59, 3295-3300 (1999).

24. Hacker, B., Welte, S., Rempe, G. \& Ritter, S. A photon-photon quantum gate based on a single atom in an optical resonator. Nature 536, 193-196 (2016).

25. Tiarks, D., Schmidt-Eberle, S., Stolz, T., Rempe, G. \& Dürr, S. A photon-photon quantum gate based on Rydberg interactions. Nat. Phys. 15, 124-126 (2019).
26. Wang, X. W., Zhang, D. Y., Tang, S. Q. \& Xie, L. J. Nondestructive Greenberger-Horne-Zeilinger-state analyzer. Quantum Inf. Process. 12, 1065-1075 (2013).

27. Duan, L. M. \& Kimble, H. J. Scalable photonic quantum computation through cavity-assisted interactions. Phys. Rev. Lett. 92, 127902 (2004).

28. Reiserer, A. A., Kalb, N., Rempe, G. \& Ritter, S. A quantum gate between a flying optical photon and a single trapped atom. Nature 508, 237-240 (2014).

29. Lundeen, J. S. et al. Tomography of quantum detectors. Nat. Phys. 5, 27-30 (2009).

30. Bouwmeester, D., Pan, J.-W., Daniell, M., Weinfurter, H. \& Zeilinger, A. Observation of three-photon Greenberger-Horne-Zeilinger entanglement. Phys. Rev. Lett. 82, 1345-1349 (1999).

Publisher's note Springer Nature remains neutral with regard to jurisdictional claims in published maps and institutional affiliations.

\section{(c) (i)}

Open Access This article is licensed under a Creative Commons

Attribution 4.0 International License, which permits use, sharing, adaptation, distribution and reproduction in any medium or format, as long as you give appropriate credit to the original author(s) and the source, provide a link to the Creative Commons license, and indicate if changes were made. The images or other third party material in this article are included in the article's Creative Commons license, unless indicated otherwise in a credit line to the material. If material is not included in the article's Creative Commons license and your intended use is not permitted by statutory regulation or exceeds the permitted use, you will need to obtain permission directly from the copyright holder. To view a copy of this license, visit http://creativecommons. org/licenses/by/4.0/.

(c) The Author(s) 2021 


\section{Methods}

The two single-sided Fabry-Pérot resonators at nodes 1 and 2 are each made of two mirrors separated by $0.5 \mathrm{~mm}$ with transmissions of $T_{1}=(3 \mathrm{ppm}, 92 \mathrm{ppm})$ and $T_{2}=(4 \mathrm{ppm}, 101 \mathrm{ppm})$, respectively. The relevant cavity quantum electrodynamics parameters are $g, \kappa, \gamma=2 \pi \times(7.6,2.5,3.0) \mathrm{MHz}$ and $g, \kappa, \gamma=2 \pi \times(7.6,2.8,3.0) \mathrm{MHz}$ for nodes 1 and 2, respectively, where $g, 2 \kappa$ and $2 \gamma$ indicate the atom-cavity coupling strength, the cavity linewidth and the atomic decay rate from the state $|e\rangle$, respectively. Both nodes operate in the strong-coupling regime where $g>\kappa, \gamma$.

At each node, we can prepare any atomic qubit state and detect it in any basis. This is achieved by using a pair of lasers, which can drive coherent Rabi oscillations between the states $\left|\downarrow_{z}\right\rangle$ and $\left|\uparrow_{z}\right\rangle$ via a two-photon Raman process ${ }^{24}$. In the presented experiments, a $\pi / 2$ rotation is performed within $4 \mu \mathrm{s}$. Slight intensity fluctuations and the finite bandwidth of the Raman pulse limit the state preparation fidelity to $98.5 \%$. The atomic state is measured using light resonant with the $\left|\uparrow_{z}\right\rangle \leftrightarrow|e\rangle$ transition, which allows us to discriminate between states $\left|\uparrow_{z}\right\rangle$ and $\left|\downarrow_{z}\right\rangle$ in $<5 \mu$ s with a fidelity of $>99.8 \%$ by collecting the fluorescence light through the cavity mode. The average qubit detection fidelity in different bases is then $98.3 \%$, which includes a preceding Raman pulse of given amplitude and phase.

At node 1, light emerges from a single-mode fibre, is reflected on a low-reflectivity (1.5\%) beam splitter employed as a circulator, and impinges on the first cavity. The overlap between the fibre transversal mode and the cavity mode is measured to be $92 \%$. Upon reflection, light passes through an acousto-optical modulator, which serves as a fast optical-path switch that separates atomic state-detection fluorescence light from the spatial mode of the fibre connecting the two nodes. The latter is stabilized at regular time intervals using piezoelectric fibre squeezers ${ }^{31}$ to avoid polarization fluctuations between the nodes. It has $95 \%$ intrinsic transmission at the photon wavelength $(780 \mathrm{~nm}), 67 \%$ transversal mode matching with the resonator at node 1 , and is connected to a fibre-based optical circulator, which is used to couple the light to the cavity at node 2 and to collect it upon reflection. Including the circulator transmission ( $80 \%$ for one passage), this leads to $51 \%$ overall optical losses between the two nodes. The mode matching between the cavity at node 2 and the circulator is measured to be $98 \%$. After being collected by the fibre circulator, the light is coupled out to free space and sent to a combination of waveplates and a polarizing beam splitter. At each port of the beam splitter, two fibres collect the light and guide it to two superconducting nanowire single-photon detectors (detector efficiency of $\eta \approx 90 \%$ at $\lambda=780 \mathrm{~nm}$ ). Including the second passage of the circulator, this leads to an overall detection efficiency of $50 \%$ with a dark-count rate of $9 \mathrm{~Hz}$.

At each node, a successful loading of an atom is heralded by imaging scattered cooling light with an objective $(\mathrm{NA}=0.4)$ onto an electron-multiplying charge-coupled device camera. When a single atom is present in each of the nodes, a digital signal triggers the experimental sequence, which runs at a repetition rate of $1 \mathrm{kHz}$. It starts with a $200-\mu$ s-long optical pumping phase to prepare both atoms in the $\left|\uparrow_{z}\right\rangle$ state, after which the atomic qubit states are initialized via a Raman pulse. The main protocol follows with the two ancillary Gaussian laser pulses interleaved by a $\pi / 2$ rotation on both atoms and it ends with the atomic state detection. Between the Raman state initialization pulse and the state detection, the overall protocol lasts $36 \mu$ s.

\section{Data availability}

The data that support the findings of this study are available in Zenodo with the identifier https://doi.org/10.5281/zenodo.4604775.

\section{References}

31. Rosenfeld, W. et al. Towards long-distance atom-photon entanglement. Phys. Rev. Lett. 101, 260403 (2008).

\section{Acknowledgements}

We acknowledge fruitful discussions with S. Dürr and L. Knips. This work was supported by the Bundesministerium für Bildung und Forschung via the Verbund Q.Link.X (16KIS0870), by the Deutsche Forschungsgemeinschaft under Germany's Excellence Strategy - EXC-2111 - 390814868, and by the European Union's Horizon 2020 research and innovation programme via the project Quantum Internet Alliance (QIA, GA no. 820445). E.D. acknowledges support by the Cellex-ICFO-MPQ postdoctoral fellowship programme.

\section{Author contributions}

All authors contributed to the experiment, analysis of the results and writing of the manuscript.

\section{Competing interests}

The authors declare no competing interests.

\section{Additional information}

Supplementary information The online version contains supplementary material available at https://doi.org/10.1038/s41566-021-00802-1.

Correspondence and requests for materials should be addressed to E.D.

Peer review information Nature Photonics thanks the anonymous reviewers for their contribution to the peer review of this work.

Reprints and permissions information is available at www.nature.com/reprints. 